EUROPEAN JOURNAL OF PURE AND APPLIED MATHEMATICS

Vol. 12, No. 2, 2019, 432-447

ISSN 1307-5543 - www.ejpam.com

Published by New York Business Global

\title{
Monotone iterative technique and Ulam-Hyers stability analysis for nonlinear fractional order differential equations with integral boundary value conditions
}

\author{
Sajjad Ali ${ }^{1}$, Kamal Shah ${ }^{2, *}$, Hassan Khan ${ }^{1}$, Muhammad Arif ${ }^{1}$, Shahid Mahmood ${ }^{3}$ \\ 3 Sarhad University of Science and IT, Peshawar, Khyber Pakhtunkhwa, Pakistan \\ 1 Abdul Wali Khan University Mardan, Khyber Pakhtunkhwa, Pakistan \\ 2 University of Malakand, Dir(L), Khyber Pakhtunkhwa, Pakistan
}

\begin{abstract}
In this manuscript, the monotone iterative scheme has been extended to the nature of solution to boundary value problem of fractional differential equation that consist integral boundary conditions. In this concern, some sufficient conditions are developed in this manuscript. On the base of sufficient conditions, the monotone iterative scheme combined with lower and upper solution method for the existence, uniqueness, error estimates and various view plots of the extremal solutions to boundary value problem of nonlinear fractional differential equations have been studied. The obtain results have clarified the nature of the extremal solutions. Further, the Ulam-Hyers and Ulam-Hyers-Rassias stability have been investigated for the considered problem. Two illustrative examples of the BVP of the nonlinear fractional differential equations have been provided to justify our contribution.
\end{abstract}

2010 Mathematics Subject Classifications: 26A33, 34A08, 34A45

Key Words and Phrases: Nonlinear Fractional differential equations, Iterative technique, Maximal and minimal solutions, Uniqueness and existence

\section{Introduction}

In the last few decades, the class of nonlinear fractional differential equations (NFDEs) has been attracted the attentions of researchers very well. The area devoted to study NFDEs has attracted many researcher in almost every field of science. We refer some papers in $[1-8]$ about the applications. Various aspects like existence theory for the aforesaid equations has been considered in plenty of research papers, see $[9-12,17,18]$ for existence and uniqueness of solutions to NFDEs.

* Corresponding author.

DOI: https://doi.org/10.29020/nybg.ejpam.v12i2.3407

Email addresses: sajjad_ali@sbbu.edu.pk (S. Ali),

kamalshah408@gmail.com (K. Shah), hassanmath@awkum.edu.pk (H. Khan),

marifmaths@awkum.edu.pk (M. Arif), shahidmahmood757@gmail.com (S. Mahmood) 
The iterative technique with the method of upper and lower solutions has gained much attention of most of the researcher in last few decades. It is very useful tools for the existence and approximation of solutions to the initial and boundary value problems. This scheme is well studied for the intial value problems, we refer to see the work in [13-16, 19-24]. However, The study of the scheme is on intial stage for integral boundary value problem. A few authors have its study for NFDEs with boundary conditions. for instance, Khan [25] have considered it for the following class of FDEs

$$
\left\{\begin{array}{l}
{ }^{C} D^{p} z(t)+h(t, z(t))=0, \quad 1<p<2, \\
\left.z^{\prime}(t)\right|_{t=0}=0, z(1)=\delta z(t), 4
\end{array}\right.
$$

where $\delta, t \in(0,1)$.

The aforementioned method has also been applied to the study of a coupled system of the NFDEs with three point boundary conditions by kamal[26] and his co-author.

Recently, the investigation of stability is also a key research area for the the development of the fractional calculus. It an important field of fractional calculus. In this concern many researchers have introduced numerous type of stability including exponential stability, Lyapunov stability, Ulam type stability analysis etc. Among them, the Ulam type stability has attracted the attention of most of the authors. The Ulam type stability has been well investigated for classical differential equations. In last few years, for initial value problems of FDEs, the Ulam type stability has been well investigated in many articles for detail see [27-31]. But, as far we know, its study is very limited for BVPs of FDEs. Therefore inspired from the aforesaid work, we study the following NFDEs with integral boundary value conditions as

$$
\begin{aligned}
& { }^{C} D^{p} z(s)+Q(s, z(s))=0, s \in[0,1], 2<p \leq 3, \\
& z(0)=\left.z^{\prime \prime}(s)\right|_{s=0}=0, z(1)=\delta \int_{0}^{1} z(s) d s,
\end{aligned}
$$

where ${ }^{C} D^{p}$ denotes Caputo derivative, $Q: I \times \mathbb{R} \rightarrow \mathbb{R}, 2<p \leq 3$ and $0<\delta<2$, $z \in A C^{2}[0,1]$.

In comparison to the work $[10,11,25,26]$, we contribute the improvement of iterative scheme together with upper and lower solution to study existence, uniqueness, error estimates and plotting of the iterative extremal solutions of nonlinear fractional differential equations with integral boundary conditions (1). In addition, we investigate stability analysis for the solution of nonlinear fractional differential equations which not established in the work of $[10,11,25,26]$. Two illustrative examples of problem (1) have been considered to demonstrate our existence theory. Behavior of the upper and lower solutions of proposed examples are plotted as Figure 1 and 2 via using Matlab which have not been done in the work of cited above.

\section{Preliminaries}

We provide the following few preliminary results, we refer to see $[2-5,17-19]$. 
Definition 1. The Riemann-Liouville integral of fractional order $\delta \in \mathbb{R}_{+}$of a function $\phi$ is defined by

$$
I^{p} \phi(s)=\frac{1}{\Gamma(p)} \int_{0}^{s}(s-\gamma)^{p-1} \phi(\gamma) d \gamma
$$

provided the right side exits.

Definition 2. [3] The Caputo fractional order derivative of order $p$ of function $\phi$ is defined by

$$
{ }^{C} D^{p} \phi(s)=\frac{1}{\Gamma(n-p)} \int_{0}^{s}(s-\gamma)^{n-p-1} \phi^{(n)}(\gamma) d \gamma,
$$

provided that integral on the right is pointwise defined on $(0, \infty)$, where $n=\lfloor p\rfloor+1$ and $\lfloor p\rfloor$ denotes the greatest integer which is less than or equal to the real number $p$.

Definition 3. [33] Let $V=C[0,1]$ be the Banach space endowed with $\|z\|=\max _{s \in[0,1]}|z(s)|$ which satisfies the partially ordering and let $U=\left[z_{1}, z_{2}\right]$ with $z_{1} \leq z_{2}$ be a set such that $U \subset V$,

Then the operator $T: U \rightarrow V$ is known as increasing function if for each $z_{1}, z_{2} \in U$ and $z_{1} \leq z_{2}$ gives $T z_{1} \leq T z_{2}$. The operator $T$ is known as decreasing function if for each $z_{1}, z_{2} \in U$ and $z_{1} \leq z_{2}$ gives $T z_{1} \geq T z_{2}$.

Definition 4. [33] Let $(I-T) z_{1} \leq 0$, then $z_{1} \in U$ is known as a lower solution of $(I-T) z=0$ and $(I-T) z_{2} \geq 0$, then $z_{2} \in U$ is known as an upper solution of $(I-T) z=0$.

Lemma 1. [18] Let $W$ be Banach Space with satisfying partially order and $z_{n}, y_{n} \in U$ so that $z_{n} \leq y_{n}, n \in Z^{+}$. If $z_{n} \rightarrow z$ and $y_{n} \rightarrow y$, then $z \leq y$.

Assume that

$\left(C_{1}\right) Q:[0,1] \times \mathbb{R} \rightarrow \mathbb{R}$ satisfies Caratheodory conditions;

$\left(C_{2}\right) Q(s, z)$ is increasing with respect to $z$ for each $s \in[0,1]$;

$\left(C_{3}\right)$ There is a constant $B>0$ so that $0 \leq Q\left(s, z_{1}(s)\right)-Q\left(s, z_{2}(s) \leq B\left(z_{1}-z_{2}\right)\right.$.

Lemma 2. [32] Let $p>0$, then

$$
z(s)=\sum_{i=0}^{\lfloor p\rfloor} \frac{z^{i}(0)}{i !} s^{i}
$$

is the solution of fractional differential equation

$$
{ }^{C} D^{p} z(s)=0 .
$$

Lemma 3. [32] Let $p>0$, then in view of Definition 24 and Lemma 2, the solution of the solution of fractional differential equation

$$
{ }^{C} D^{p} z(s)=y(s)
$$

is given by

$$
z(s)=I^{p} y(s)+\sum_{i=0}^{\lfloor p\rfloor} \frac{z^{i}(0)}{i !} s^{i} .
$$




\section{Iterative Solutions}

This portion of the manuscript is devoted to existence and uniqueness of extremal solutions to the considered BVP of the nonlinear FDE (1). Further, we study the iterative technique coupled with method of the lower and upper solutions for the approximation and error estimates to the extremal solution of (1). We also investigate the various type stabilities like Ulam-Hyers stability, generalized Ulam-Hyers stability, Ulam-Hyers-Rassias stability and generalized Ulam-Hyers-Rassias stability for the considered BVP. First, we convert our considered problem into the integral equation.

Lemma 4. If $Q \in C[0,1]$, then the linear problem

$$
\begin{aligned}
& { }^{C} D^{p} z(s)+Q(s)=0, s \in[0,1], 2<p \leq 3, \\
& z(0)=\left.z^{\prime \prime}(s)\right|_{s=0}=0, z(1)=\delta \int_{0}^{1} z(s) d s .
\end{aligned}
$$

has a solution defined by

$$
z(s)=\int_{0}^{1} R(t, \gamma) Q(\gamma) d \gamma
$$

where $R(s, \gamma)$ is defined by

$R(s, \gamma)=\frac{1}{(2-\delta) \Gamma(p+1)}\left\{\begin{array}{lr}2 s(1-\gamma)^{p-1}(p-\delta+\delta \gamma)-(2-\delta) p(s-\gamma)^{p-1}, & 0 \leq \gamma \leq s \leq 1 \\ 2 s(1-\gamma)^{p-1}(p-\delta+\delta \gamma), & 0 \leq s \leq \gamma \leq 1\end{array}\right.$

Proof. We may apply a well known Lemma 3 to reduce the equation (6) to equivalent integral form

$$
z(s)=-I^{p} Q(s)+\sum_{i=0}^{2} \frac{z^{(i)}(0)}{i !} s^{i}=-\int_{0}^{s} \frac{(s-\gamma)^{p-1}}{\Gamma(p)} Q(\gamma) d \gamma+\sum_{i=0}^{2} \frac{z^{(i)}(0)}{i !} s^{i} .
$$

By considering the given conditions $z(0)=z^{\prime \prime}(0)=0, z(1)=\delta \int_{0}^{1} z(s) d s$, we can obtain that

$$
z(s)=-\int_{0}^{s} \frac{(s-\gamma)^{p-1}}{\Gamma(p)} Q(\gamma) d \gamma+s \int_{0}^{1} \frac{(1-\gamma)^{p-1}}{\Gamma(p)} Q(\gamma) d \gamma+t \delta \int_{0}^{1} z(s) d s .
$$


Let say

$$
\begin{aligned}
& A=\int_{0}^{1} z(s) d s \\
& A=-\int_{0}^{1} \int_{0}^{s} \frac{(s-\gamma)^{p-1}}{\Gamma(p)} Q(\gamma) d \gamma d s+\int_{0}^{1} \int_{0}^{1} s \frac{(1-\gamma)^{p-1}}{\Gamma(p)} Q(\gamma) d \gamma d s+\delta A \int_{0}^{1} s d s \\
& A=-\frac{2}{(2-\delta)} \int_{0}^{1} \frac{(1-\gamma)^{p}}{p \Gamma(p)} Q(\gamma) d \gamma+\frac{1}{(2-\delta)} \int_{0}^{1} \frac{(1-\gamma)^{p-1}}{\Gamma(p)} Q(\gamma) d \gamma .
\end{aligned}
$$

Then equation (3.5) implies

$$
\begin{gathered}
z(s)=-\int_{0}^{s} \frac{(s-\gamma)^{p-1}}{\Gamma(p)} Q(\gamma) d \gamma+\int_{0}^{1} \frac{s(1-\gamma)^{p-1}}{\Gamma(p)} Q(\gamma) d \gamma-\frac{2 \delta}{(2-\delta)} \int_{0}^{1} \frac{s(1-\gamma)^{p}}{p \Gamma(p)} Q(\gamma) d \gamma \\
+\frac{\delta}{(2-\delta)} \int_{0}^{1} \frac{t(1-\gamma)^{p-1}}{\Gamma(p)} Q(\gamma) d \gamma \\
z(s)=\int_{0}^{1} K(s, \gamma) Q(\gamma) d \gamma
\end{gathered}
$$

Clearly, for all $s, \gamma \in[0,1]$, we have $R(s, \gamma) \geq 0$.

Lemma 5. If we consider the Lemma \& and condition $\left(C_{1}\right)$ with $Q \in C([0,1], \mathbb{R})$, then we see that the integral equation

$$
z(s)=\int_{0}^{1} R(s, \gamma) Q(\gamma) d \gamma
$$

is the solution of problem (1), where $R(s, \gamma)$ is provided in the equation (8).

Lemma 6. For Green's function $R(s, \gamma)$, we have that

$$
\max _{s \in[0,1]} \int_{0}^{1} R(s, \gamma) d \gamma \leq \frac{2}{(2-\delta) \Gamma(p)}, s \in[0,1] .
$$

Proof. Since $R(s, \gamma) \leq \frac{2 t(1-\gamma)^{p-1}(p-\delta+\delta \gamma)}{(2-\delta) \Gamma(p+1)}$, it follows that

$$
\max _{s \in[0,1]} \int_{0}^{1} R(s, \gamma) d \gamma \leq \frac{2}{(2-\delta) \Gamma(p)} .
$$

We define the operator $T: U \rightarrow U$ by

$$
T z(s)=\int_{0}^{1} R(s, \gamma) Q(\gamma, z(\gamma)) d \gamma
$$


Then, in view of (11) and (12), we obtain the operator equation

$$
(I z-T z)(s)=0, s \in[0,1] .
$$

Let consider the condition $\left(C_{2}\right)$, then for $z_{1}, z_{2} \in U$ with $z_{1} \leq z_{2}$, we obtain

$$
\begin{aligned}
T z_{1}(s) & =\int_{0}^{1} R(s, \gamma) Q\left(\gamma, z_{1}(\gamma)\right) d \gamma \\
& \leq \int_{0}^{1} R(s, \gamma) Q\left(\gamma, z_{2}(\gamma)\right) d \gamma=T z_{2}(s)
\end{aligned}
$$

that is, $T$ is nondecreasing.

We can assume another condition which is

$\left(C_{4}\right)$ For minimal and maximal solutions $\alpha, \beta \in U$ of the equation (13), the inequality $\alpha \leq \beta$ on $[0,1]$ is obvious .

Lemma 7. Under the conditions $\left(C_{1}\right)-\left(C_{4}\right)$ the iterative solutions of operator equation (13) is the monotonic sequence which converges to the solution of equation (11).

Proof. We consider that the conditions $\left(C_{1}\right)-\left(C_{4}\right)$ hold, then we show that $T$ is continuous. For this, let $z_{1}, z_{2} \in U$, then

$$
\begin{aligned}
& \left|T z_{1}(s)-T z_{2}(s)\right| \\
& =\left|\int_{0}^{1} R(s, \gamma) Q\left(\gamma, z_{1}(\gamma)\right) d \gamma-\int_{0}^{1} R(s, \gamma) Q\left(\gamma, z_{2}(\gamma)\right) d \gamma\right| \\
& =\left|\int_{0}^{1}\left[R(s, \gamma) Q\left(\gamma, x_{1}(\gamma)\right)-R(s, \gamma) Q\left(\gamma, z_{2}(\gamma)\right)\right] d \gamma\right| \\
& \leq \int_{0}^{1} R(s, \gamma) \mid Q\left(\gamma, z_{1}(\gamma)-Q\left(\gamma, z_{2}(\gamma)\right) \mid d \gamma\right. \\
& \leq \frac{2 B}{(2-\delta) \Gamma(p)}\left\|z_{1}-z_{2}\right\| .
\end{aligned}
$$

Thus $T$ is continuous. It is easy to prove that $T$ is also uniformly bounded and equicontinuous. Thank to the Arzela Ascoli theorem's statement: "If $M$ be a family (finite or infinite) of an equi-continuous, uniformly bounded real valued functions $z$ on an interval $[0,1]$. Then $M$ contains a uniformly convergent sequence of functions $z_{n}$, converging to a function $z$ in $U$ as $n \rightarrow \infty$, where $U$ denotes the space of all continuous bounded functions on $[0,1]$. Thus any sequence in $M$ contains a uniformly bounded convergent subsequence on $[0,1]$ and consequently $M$ has a compact closure in $U$ ". We obtain $T$ is compact. Further, we may choose $z_{0}=\alpha$. then, in the view of $C_{4}$, we obtain

$$
\begin{gathered}
\alpha \leq \beta \\
z_{0} \leq \beta
\end{gathered}
$$




$$
\begin{gathered}
z_{0} \leq T z_{0} \leq T \beta \leq \beta, T \text { is increasing } \\
z_{0} \leq z_{1} \leq \beta \text { on }[0,1], \text { where } z_{1}=T z_{0} .
\end{gathered}
$$

Similarly,

$T z_{0} \leq T z_{1} \leq T \beta \leq \beta$, that is, $z_{1} \leq z_{2} \leq \beta$ on $[0,1]$, where $z_{2}=T z_{1}$.

In same way, we obtain the bounded monotone sequence $\left\{z_{n}\right\}$, that is

$$
z_{0} \leq z_{1} \leq z_{2} \leq \ldots z_{n-1} \leq z_{n} \leq \beta \text { on }[0,1]
$$

where $z_{n}=T z_{n-1}$. Then, there will be $z \in U$ such that $z_{n} \rightarrow z$ as $n \rightarrow \infty$. Hence, we obtain that $z=T z$. But $z$ is solution of the equation (11) given by

$$
z(s)=\int_{0}^{1} R(s, \gamma) Q(\gamma, z(\gamma)) d \gamma, s \in[0,1] .
$$

Let consider the condition $\left(C_{3}\right)$, then for $z, y \in U$ with $z \leq y$, we have the inequality

$$
\|T y-T z\| \leq \Delta\|y-z\|, \text { where } \Delta=\frac{2 B}{(2-\delta) \Gamma(p)} .
$$

Hence in view of (15) and (16), we get

$$
\begin{aligned}
& \left\|z_{2}-z_{1}\right\|=\left\|T z-T z_{0}\right\| \leq \Delta e_{1}, \\
& \left\|z_{3}-z_{2}\right\|=\left\|T z_{2}-T z_{0}\right\| \leq \Delta^{2} e_{1}, \\
& \left\|z_{4}-z_{3}\right\|=\left\|T z_{3}-T z_{2}\right\| \leq \Delta^{3} e_{1}, \\
& \vdots \\
& \left\|z_{n+1}-z_{n}\right\|=\left\|T z_{n}-T z_{0}\right\| \leq \Delta^{n} e_{1} .
\end{aligned}
$$

Therfore, we can obtain

$\left\|z_{m+n}-z_{n}\right\| \leq\left\|z_{n+p}-z_{n+p-1}\right\|+\left\|z_{n+m-1}-z_{n+m-2}\right\|+\ldots\left\|z_{n+1}-z_{n}\right\| \leq \Delta^{n} \frac{1-\Delta^{m}}{1-\Delta} e_{1}$.

where $m, n$ are positive integers and $\Delta<1$. Now if $n \rightarrow \infty$, then we obtain that $\| z_{m+n}-$ $z_{n} \| \rightarrow 0$. Hence $\left\{z_{n}\right\}$ is Cauchy sequence in $U$. Thus $z^{*}(s)=\lim _{n \rightarrow \infty} z_{n}(s)$. Therefore, we obtain that $T z^{*}=z^{*}$. Let $m \rightarrow \infty$ in the equation (17), then estimate of error for the minimal solution is provided by

$$
e_{n}=\left\|z^{*}-z_{n}\right\| \leq \frac{\Delta^{n}}{1-\Delta} e_{1}, \text { where } e_{1}=\left\|z_{1}-z_{0}\right\| .
$$

Remark. If we consider that $z_{0}=\beta$, then we have that $\left\{z_{n}\right\}$ is Cauchy sequence such that $z_{0} \geq z_{1} \geq z_{2} \geq \ldots z_{n-1} \geq z_{n} \geq \alpha$ on $[0,1]$, 
which converges to the solution of the equation (11). Hence, we obtain corresponding estimate of error for the maximal solutions which is provided by $e_{n}^{*}=\left\|z_{n}^{*}-\bar{z}^{*}\right\| \leq$ $\frac{\Delta^{n}}{1-\Delta} e_{1}^{*}$, where $e_{1}^{*}=\left\|z_{0}^{*}-z_{1}^{*}\right\|$. In the view of Lemma 7 , we can fix the iterative schemes for NFDE (1) as

$$
\begin{aligned}
& z_{m}(s)=\int_{0}^{1} R(s, \gamma) Q\left(\gamma, z_{m-1}\right) d \gamma, m \geq 1, \\
& z_{m}^{*}(s)=\int_{0}^{1} R(s, \gamma) Q\left(\gamma, z_{m-1}^{*}\right) d \gamma, m \geq 1,
\end{aligned}
$$

from which, we obtain $z^{*}(s)=\lim _{m \rightarrow \infty} z_{m}(s)$

$\bar{z}^{*}(s)=\lim _{m \rightarrow \infty} z_{m}^{*}(s)$.

Theorem 1. Under the assumptions $\left(C_{1}\right),\left(C_{2}\right)$ and $\left(C_{3}\right)$ with $\Delta<1$, there exist unique lower and upper solutions to the problem (1).

Proof. Proof is similar as given in [33].

\section{Ulam type stability}

In this section, we develop sufficient conditions for the Ulam stability analysis of the solutions to the considered BVP of FDEs (1). The required definitions and lemmas can be found in [27-31, 34-36].

Definition 5. If, we have $\mathbf{c}_{K} \in \mathbb{R}^{+}$and for every $\varepsilon>0$ such that for any solution $z \in A C^{2}[0,1]$ of

$$
\left|{ }^{C} D^{p} z(s)+Q(s, z(s))\right| \leq \varepsilon, s \in[0,1],
$$

there exists $v \in A C^{2}[0,1]$ is a unique solution of equation (1) such that

$$
|z(s)-v(s)| \leq \mathbf{c}_{K} \varepsilon, s \in[0,1] .
$$

Then BVP (1) is said to be Ulam-Hyers stable.

Definition 6. If, we have $\varphi \in C\left(\mathbb{R}^{+}, \mathbb{R}^{+}\right), \varphi(0)=0$ and for every $\varepsilon>0$ so that for any solution $z \in A C^{2}[0,1]$ of

$$
\left|{ }^{C} D^{p} z(s)+Q(s, z(s))\right| \leq \varepsilon, s \in[0,1],
$$

there exists $v \in A C^{2}[0,1]$ is a unique solution of the considered problem (1) such that

$$
|z(s)-v(s)| \leq \phi(s) \varepsilon, s \in[0,1] .
$$

Then BVP of FDEs (1) is said to be generalized Ulam-Hyers stable. 
Definition 7. If, we have $\phi \in C\left([0,1], \mathbb{R}^{+}\right), \mathbf{c}_{K}>0$ and for every $\varepsilon>0$ such that for any solution $z \in A C^{2}[0,1]$ of inequality

$$
\left|{ }^{C} D^{p} z(s)+Q(s, z(s))\right| \leq \varepsilon \phi(s), s \in[0,1],
$$

there exists $v \in A C^{2}[0,1]$ is a unique solution of considered problem (1) such that

$$
|z(s)-v(s)| \leq \mathbf{c}_{K} \varepsilon \phi(s), s \in[0,1] .
$$

Then BVP(1) is said to be the Ulam-Hyers-Rassias stable with respect to $\phi \in C\left([0,1], \mathbb{R}^{+}\right)$.

Definition 8. If, we have $\phi \in C([0,1], \mathbb{R})$ and $\mathbf{c}_{\phi} \in \mathbb{R}^{+}$so that for any solution $z \in$ $A C^{2}[0,1]$ of inequality

$$
\left|{ }^{C} D^{p} z(s)+Q(s, z(s))\right| \leq \phi(s), s \in[0,1],
$$

there exists $v \in A C^{2}[0,1]$ is a unique solution of considered problem (1) so that

$$
|z(s)-v(s)| \leq \mathbf{c}_{\phi} \phi(s), s \in[0,1] .
$$

Then BVP(1) is said to be generalized Ulam-Hyers-Rassias stable with respect to $\phi \in$ $C([0,1], \mathbb{R})$.

Remark 1. Let $z \in A C^{2}[0,1]$ is the solution of inequality (19) whenever, there exists the function $\psi \in C([0,1], \mathbb{R})$ (dependent on $z$ ), so that

(i) ${ }^{C} D^{p} z(s)+Q(s, z(s))=\psi(s), s \in[0,1]$;

(ii) $|\psi(s)| \leq \varepsilon$, for all $s \in[0,1]$.

Theorem 2. If consider the conditions $\left(C_{2}\right),\left(C_{3}\right)$ coupled with $\delta<1$, then the UlamHyers stability results for the solutions of $B V P(1)$ are obtained which further implies that the solutions of BVP (1) are generalized Ulam-Hyers stable.

Proof. Let consider the conditions $\left(C_{2}\right),\left(C_{3}\right)$ coupled with $\delta<1$. Let $z \in A C^{2}[0,1]$ be any solution of BVP (1). Then Using the condition $(i)$ of the Remark 1 for $z \in A C^{2}[0,1]$, which is

$$
{ }^{C} D^{p} z(s)+Q(s, z(s))=\psi(s), \text { for all } s \in[0,1], 2<p \leq 3 .
$$

Thank to Lemma 4 , we obtain

$$
z(s)=\int_{0}^{1} R(s, \gamma) Q(\gamma, z(\gamma)) d \gamma+\int_{0}^{1} R(s, \gamma) \psi(\gamma) d \gamma, \text { where } \psi \in C([0,1], \mathbb{R}) .
$$

Which yields

$$
\left|z(s)-\int_{0}^{1} R(s, \gamma) Q(\gamma, z(\gamma)) d \gamma\right| \leq \frac{2 \varepsilon}{(2-\delta) \Gamma(p)}, s \in[0,1] .
$$


Let $v \in A C^{2}[0,1]$ be a unique solution of BVP (1).Then

$$
\begin{aligned}
& |z(s)-v(s)| \\
= & \left|z(s)-\int_{0}^{1} R(s, \gamma) Q(\gamma, v(\gamma)) d \gamma\right| \\
= & \left|z(s)-\int_{0}^{1} R(s, \gamma) Q(\gamma, z(\gamma)) d \gamma+\int_{0}^{1} R(s, \gamma) Q(\gamma, z(\gamma)) d \gamma-\int_{0}^{1} R(s, \gamma) Q(\gamma, v(\gamma)) d \gamma\right| \\
\leq & \left|z(s)-\int_{0}^{1} R(s, \gamma) Q(\gamma, z(\gamma)) d \gamma\right|+\int_{0}^{1}|R(s, \gamma)| Q(\gamma, z(\gamma))-Q(\gamma, v(\gamma)) \mid d \gamma .
\end{aligned}
$$

By using the inequality (22), we obtain that

$$
\begin{aligned}
& \|z-v\| \leq \frac{2 \varepsilon}{(2-\delta) \Gamma(p)}+\frac{2 B}{(2-\delta) \Gamma(p)}\|z-v\|, \\
& \text { hence we have }\|z-v\| \leq \mathbf{c}_{K} \varepsilon \text {, where } \mathbf{c}_{K}=\frac{2}{(2-\delta) \Gamma(p)-2 B}>0 \text {. }
\end{aligned}
$$

Hence the solutions of BVP (1) are Ulam-Hyers stable. When, we substitute $\phi(\varepsilon)=\mathbf{c}_{K} \varepsilon$, $\phi(0)=0$. Then consequently generalized Ulam-Hyers stability for the solutions of BVP (1) are obtained.

Remark 2. Let $z \in A C^{2}[0,1]$ is the solution of inequality (20) whenever there exists the function $\psi \in C([0,1], \mathbb{R})$ (dependent on $z$ ) so that

(i) ${ }^{C} D^{p} z(s)+Q(s, z(s))=\psi(s), s \in[0,1]$;

(ii) $|\psi(s)| \leq \varepsilon \phi(s)$, for all $s \in[0,1]$, where $\phi \in C([0,1], \mathbb{R})$.

By Using the Remark 2, Let $z \in A C^{2}[0,1]$ be any solution of BVP (1) such that

$$
{ }^{C} D^{p} z(s)+Q(s, z(s))=\psi(s), s \in[0,1] .
$$

Then, thank to Lemma 4, we have

$$
z(s)=\int_{0}^{1} R(s, \gamma) Q(\gamma, z(\gamma)) d \gamma+\int_{0}^{1} R(s, \gamma) \psi(\gamma) d \gamma, \text { where } \psi \in C([0,1], \mathbb{R})
$$

which yields

$$
\left|z(s)-\int_{0}^{1} R(s, \gamma) Q(\gamma, z(\gamma)) d \gamma\right| \leq \frac{2 \varepsilon}{(2-\delta) \Gamma(p)} \phi(s), s \in[0,1] .
$$

Theorem 3. If consider the conditions $\left(C_{2}\right),\left(C_{3}\right)$ coupled with $\delta<1$, then the UlamHyers-Rassias stability results for the solutions of BVP (1) are obtained which further implies that the solutions of BVP (1) are generalized Ulam-Hyers-Rassias stable. 
Proof. Let consider the conditions $\left(C_{2}\right),\left(C_{3}\right)$ coupled with $\delta<1$. If $v \in A C^{2}[0,1]$ is the unique solution of BVP (1) and $z \in A C^{2}[0,1]$ be any solution of BVP (1) which is also the solution (24). Then, we have

$$
\begin{aligned}
& |v(s)-z(s)| \\
= & \left|\int_{0}^{1} R(s, \gamma) Q(\gamma, v(\gamma)) d \gamma-z(s)\right| \\
= & \left|\int_{0}^{1} R(s, \gamma) Q(\gamma, v(\gamma)) d \gamma-\int_{0}^{1} R(s, \gamma) Q(\gamma, z(\gamma)) d \gamma+\int_{0}^{1} R(s, \gamma) Q(\gamma, z(\gamma)) d \gamma-z(s)\right| \\
\leq & \int_{0}^{1}|R(s, \gamma)| Q(\gamma, v(\gamma))-Q(\gamma, z(\gamma))|d \gamma+| z(s)-\int_{0}^{1} R(s, \gamma) Q(\gamma, z(\gamma)) d \gamma \mid .
\end{aligned}
$$

Which implies that

$$
\begin{aligned}
& \|v-z\| \leq \frac{2 B}{(2-\delta) \Gamma(p)}\|v-z\|+\frac{2 \varepsilon \phi(s)}{(2-\delta) \Gamma(p)}, \\
& \text { from which we get }\|v-z\| \leq \varepsilon \mathbf{c}_{K} \phi(s) \text {, where } \mathbf{c}_{K}=\frac{2}{(2-\delta) \Gamma(p)-2 B}>0 \text {. }
\end{aligned}
$$

Therefore the solutions of BVP (1) are Ulam-Hyers-Rassias stable. When, we substitute $\varphi(\varepsilon)=\mathbf{c}_{K} \varepsilon \phi(s), \varphi(0)=0$. Then consequently generalized Ulam-Hyers-Rassias stability for the solutions of BVP (1) are obtained.

\section{Examples}

In the concern section, we provide two examples of concern problem (1). We find iterative solution for the extremal solution of the considered examples. We also provide error estimates for the extremal solutions. Furthermore, stability analysis and error estimates for the solution of examples of the problem (1) is also our concern. In addition, we provide various plots view of every example of problem (1).

\section{Example 1.}

$$
\left\{\begin{array}{l}
{ }^{C} D^{\frac{5}{2}} z(s)+Q(s, z(s))=0, s \in[0,1], \\
z(0)=\left.z^{\prime \prime}(s)\right|_{s=0}=0, z(1)=\frac{1}{2} \int_{0}^{1} z(s) d s .
\end{array}\right.
$$

Where $Q(s, z(s))=\sqrt{z(s)}+\sin ^{2}[t \exp (z(s))]+\log [1+z(s)]$. Let say $n=4$ is large enough.Then, approximate minimal and maximal solutions are given by

$$
\begin{aligned}
& z(s)=z_{4}(s)=\int_{0}^{1} R(s, \gamma) Q\left(\gamma, z_{3}(\gamma)\right) d \gamma \\
& z^{*}(s)=z_{4}^{*}(s)=\int_{0}^{1} R(s, \gamma) Q\left(\gamma, z_{3}^{*}(\gamma)\right) d \gamma
\end{aligned}
$$


Let $B=0.00001$ and the lower and upper solutions of $B V P(1)$ are $z_{0}=-0.2$, and $z_{0}^{*}=$ 0.2 respectively. Then we have $\Delta=\frac{2 B}{(2-\delta) \Gamma(p)}=0.000010030<1$. Therefore, the error estimates are:

$e_{3}=\left\|z(s)-z_{3}(s)\right\| \leq \frac{\Delta^{3}}{1-\Delta} \times e_{1} \leq 1.00904832686 \times 10^{-15} \max _{s \in[0,1]}\left|z_{1}(s)+0.2\right| \simeq 2.22668549047 \times 10^{-16}$,
$e_{3}^{*}=\left\|z(s)-z_{3}^{*}(s)\right\| \leq \frac{\Delta^{3}}{1-\Delta} \times e_{1}^{*} \leq 1.00904832686 \times 10^{-15} \max _{s \in[0,1]}\left|0.2-z_{1}^{*}(s)\right| \simeq 2.01809665372 \times 10^{-16}$.

The maximal and minimal solutions of the Example (1) are plotted in the Figure 1.

Clearly, the condition $\Delta<1$ is sufficient for various kinds of Ulam stabilities for the extremal solution of the Example (1). The stability of extremal solutions is also obvious from plots in the given Figure 1.
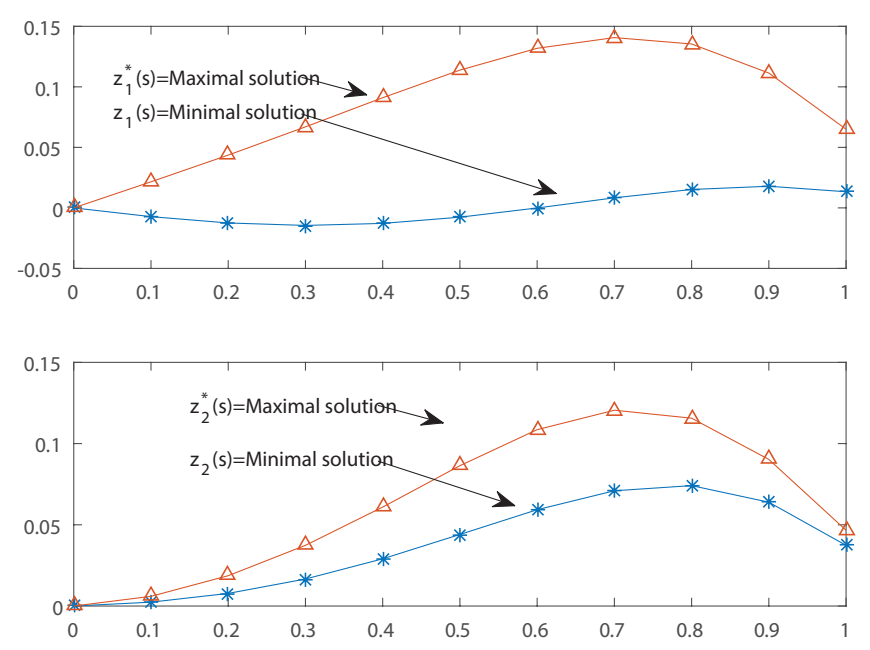

Figure 1 A line graph of the extremal solutions of the Example 1.

\section{Example 2.}

$$
\left\{\begin{array}{l}
{ }^{C} D^{\frac{11}{4}} z(s)+Q(s, z(s))=0, s \in[0,1], \\
z(0)=\left.z^{\prime \prime}(s)\right|_{s=0}=0, z(1)=\frac{1}{10} \int_{0}^{1} z(s) d s .
\end{array}\right.
$$

Where $Q(s, z(s))=t \sqrt{z(s)}+\exp (z(s)$. Let say $n=4$ is large enough. Then, approximate minimal and maximal solutions are given by

$$
\begin{aligned}
& z(s)=z_{4}(s)=\int_{0}^{1} R(s, \gamma) Q\left(\gamma, z_{3}(\gamma)\right) d \gamma \\
& z^{*}(s)=z_{4}^{*}(s)=\int_{0}^{1} R(s, \gamma) Q\left(\gamma, z_{3}^{*}(\gamma)\right) d \gamma
\end{aligned}
$$


Let $B=0.00001$ and the lower and upper solutions of $B V P(2)$ are $z_{0}=-0.5$, and $z_{0}^{*}=0.5$ respectively. Then, we have $\Delta=\frac{2 B}{(2-\delta) \Gamma(p)}=6.5448 e^{-06}<1$. Therefore, the corresponding estimates of error are:

$$
\begin{aligned}
& e_{3}=\left\|z(s)-z_{3}(s)\right\| \leq \frac{\Delta^{3}}{1-\Delta} \times e_{1} \leq 2.8034 \times 10^{-16} \max _{s \in[0,1]}\left|z_{1}(s)+0.5\right| \simeq 1.5469 \times 10^{-16}, \\
& e_{3}^{*}=\left\|z(s)-z_{3}^{*}(s)\right\| \leq \frac{\Delta^{3}}{1-\Delta} \times e_{1}^{*} \leq 2.8034 \times 10^{-16} \max _{s \in[0,1]}\left|0.5-z_{1}^{*}(s)\right| \simeq 1.4017 \times 10^{-16} .
\end{aligned}
$$

The maximal and minimal solutions of the Example (2) are plotted in the Figure 2.

Clearly, the condition $\Delta<1$ is sufficient for various kinds of Ulam stabilities for the extremal solution of the Example (2). The stability of extremal solutions is also obvious from plots in the given Figure 2.
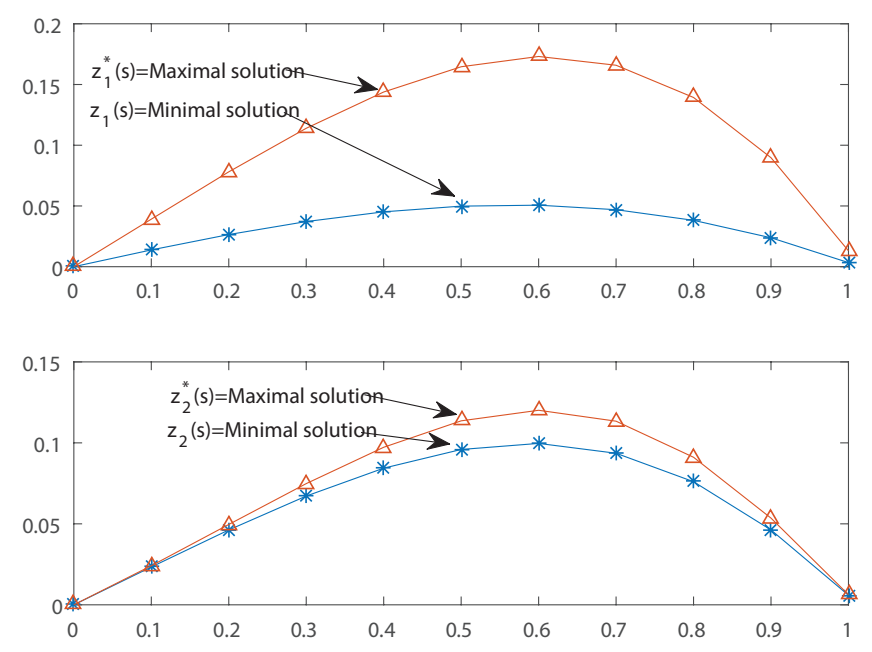

Figure. 2 A line graph of the extremal solutions of the Example 2.

\section{Conclusion}

We have successfully investigated sufficient conditions for maximal and minimal solutions using monotone iterative schemes together with upper and lower solutions method. We also provided maximum error estimates for the solutions. The obtained results were verified by plotting the graph of the illustrative problem.

\section{Acknowledgements}

The authors would like to thank the reviewers of this paper for their valuable comments on the earlier version of the paper. They would also like to acknowledge Prof. Dr. Salim ur Rehman, V.C. Sarhad University of Science and I. T, for providing excellent research 
and academic environment.

Funding Source: We remark that this work has been supported by the HEC of Pakistan, Grant No: 10039/KPK/ NRPU/R\&D/HEC/ 2017 and HED of KPK, Government of Pakistan at Grant No: HEREF-46

Authors contributions: All authors jointly worked on the results and they read and approved the final manuscript.

Conflict of interest: It is declared that no conflict of interest exist regarding this paper.

\section{References}

[1] A. A. Kilbas, O.I. Marichev and S. G. Samko. Fractional Integrals and Derivatives (Theory and Applications). Gordon and Breach, Switzerland, 1993.

[2] K. S. Miller and B. Ross. An Introduction to the Fractional Calculus and Fractional Differential Equations. Wiley, New York, 1993.

[3] I. Podlubny. Fractional Differential Equations, Mathematics in Science and Engineering. Academic Press, New York, 1999.

[4] R. Hilfer. Applications of Fractional Calculus in Physics. World Scientific, Singapore, 2000.

[5] A. A. Kilbas, H. M. Srivastava and J.J. Trujillo. Theory and Applications of Fractional Differential Equations. Elsevier, Amsterdam, 2006.

[6] M. Benchohra, J. R. Graef, and S. Hamani. Existence results for boundary value problems with nonlinear fractional differential equations. Applied Analysis, 87:851$863,2008$.

[7] R. P. Agarwal, M. Belmekki and M. Benchohra. A survey on semilinear differential equations and inclusions involving Riemann-Liouville fractional derivative. Advances in. Difference Equations, 2009:14 pages, 2009.

[8] R. P. Agarwal, M. Benchohra and S. Hamani. A survey on existence results for boundary value problems of nonlinear fractional differential equations and inclusions. Acta Applicandae Mathematicae, 109:973-1033, 2012.

[9] W.Yang. Positive solution to nonzero boundary values problem for a coupled systemof nonlinear fractional differential equations. Computer and Mathematics with Applications, 63:288-297, 2012.

[10] K.Shah and R.A. Khan. Iterative solutions to a coupled system of nonlinear fractional differential equations. Journal of Fractional Calcculus and Applications, 7(2):40-50, 2016. 
[11] K. Shah, H. Khalil and R. A. Khan. Upper and lower solutions to a coupled system of nonlinear fractional differential equations. Progress in Fractional Differntial Equations Applications, 1(1):1-10, 2016.

[12] B. Ahmad and J. J. Nieto. Existence results for a coupled system of nonlinear fractional differential equations with three-point boundary conditions. Computer and Mathematics with Applications, 58:1838-1843, 2009.

[13] G.S. Ladde, V.Lakshmikantham and A.S. Vatsala. Monotone iterative technique for nonlinear differential equations. Pitman Publishing Inc., Boston, 1985.

[14] S. Zhang. Monotone iterative method for initial value problem involving RiemannLiouville fractional derivatives. Nonlinear Analysis: Theory Methods and Applications, 71(5):2087-2093, 2009.

[15] F.A. Mcrae. Monotone iterative technique and existence results for fractional differential equations. Nonlinear Analysis: Theory Methods and Applications, 71(12):60936096, 2009.

[16] M. Al-Refai, M. Ali Hajji. Monotone iterative sequences for nonlinear boundary value problems of fractional order. Nonlinear Analysis: Theory Methods and Applications, 74(11):3531-3539, 2011.

[17] G. Wang, R.P. Agarwal, A. Cabada. Existence results and the monotone iterative technique for systems of nonlinear fractional differential equations. Applied Mathematical Letter, 25(6):1019-1024, 2012.

[18] E. Zeidler. Nonlinear Functional Analysis and its Applications, part II/B: Nonlinear Monotone Operators. Springer Verlag, New York, 1989.

[19] N.Xu,W.Liu. Iterative solutions for a coupled system of fractional differential-integral equations with two- point boundary conditions. Applied Mathematics and Computation, 244:903-911, 2014.

[20] X. Liu, M. Jia. Multiple solutions for fractional differential equations with nonlinear boundary conditions. Computer and Mathematics with Applications, 59:2880-2886, 2010 .

[21] Z. He, X. He. Monotone iterative technique for impulsive integro-differential equations with periodic boundary conditions. Computer and Mathematics with Applications, 48:73-84, 2004.

[22] C. De Coster, P. Habets. Two-point Boundary Value Problems: Lower and Upper Solutions. Elsevier, Amsterdam, 2006.

[23] F. Li, M. Jia, X. Liu, C. Li, G. Li. Existence and uniqueness of solutions of secendorder three-point boundary value problems with upper and lower solutions in the 
reverse order.Nonlinear Analysis: Theory Methods and Applications, 68:2381-2388, 2008 .

[24] F. Li, J. Sun, M. Jia. Monotone iterative method for the second-order threepoint boundary value problem with upper and lower solutions in the reversed order.Computer and Mathematics with Applications, 217:4840-4847, 2011.

[25] R. A. Khan. Existence and approximation of solutions to three-point boundary value problems for fractional differential equations. Electronic Journal of Qualitative Theory of Differential Equations, 58:1-8, 2011.

[26] Kamal shah and Rahmat Ali Khan. Iterative scheme for a coupled system of fractional-order differential equations with three-point boundary conditions. Mathematical Methods in the applied Sciences, 2016:7 pages, 2016.

[27] I. A. Rus. Ulam stabilities of ordinary differential equations in a Banach space. Carpathian J. Math., 26:103-107, 2010.

[28] T. M. Rassias. On the stability of the linear mapping in Banach spaces. Proc. Amer. Math. Soc., 72:297-300, 1978.

[29] T. M. Rassias. On the stability of functional equations and a problem of Ulam. Acta. Appl. Math., 26:23-130, 2000.

[30] M. Li, J. Wang, D. O'Regan. Existence and Ulam's stability for conformable fractional differential equations with constant coefficients. Bull. Malays. Math. Sci. Soc. 2017; doi:10.1007/s40840-017-0576-7.

[31] J. Wang, M. Fečkan, Y. Zhou. Fractional order differential switched systems with coupled nonlocal initial and impulsive conditions. Bull. Sci. math., 141:727-746, 2017.

[32] Alberto Cabada1 and Guotao Wang. Positive solutions of nonlinear fractional differential equations with integral boundary value conditions. Journal of Mathematical Analysis and Applications, 389(1):403-411, 2013.

[33] Sajjad Ali. Kamal Shah and Fahd Jarad. On stable iterative solutions for a class of boundary value problem of nonlinear fractional order differential equations. Math Meth Appl Sci. 2018:1-13, 2018.

[34] Th. M. Rassias and J. Brzdek. Functional Equations in Mathematical Analysis -In Honor of S. M. Ulam. Springer, New York, 2012.

[35] Pl. Kannappan. Functional Equations and Inequalities with Applications. Springer, 2009.

[36] Michael Th. Rassias. Solution of a functional equation problem of Steven Butler.Octogon Math. Mag., 12: 152 -153, 2004. 